\title{
Simulação de Déficit Hídrico em Diferentes Genótipos de Feijão pela Diminuição do Potencial Osmótico
}

Daiane M. Duarte, Diana C. Silva, Ednaldo C. Rocha, Helton S. Pereira \& Fabrício Rodrigues

O presente trabalho teve como objetivo simular condições de déficit hídrico em laboratório para a avaliação da germinação e desenvolvimento de diferentes genótipos de feijão. O experimento foi realizado no Laboratório da Universidade Estadual de Goiás, em que se utilizaram dez genótipos de feijão, submetidos à germinação em condições de estresse sob os níveis $0,-0,6,-1,2$ e -1,8 MPa de potencial osmótico, simulados com soluções de manitol $\left(\mathrm{C}_{6} \mathrm{H}_{14} \mathrm{O}_{6}\right)$ nas doses: 0, 44; 58; 89,17 e 133,75 g L-1. Dentre os genótipos utilizados, o cultivar Esplendor obteve melhor desempenho para as variáveis analisadas, demonstrando maior potencial adaptativo à deficiência hídrica.

Palavras Chaves: Phaseolus Vulgaris L.; Germinação; Estresse.

The present study aimed to simulate water deficit conditions, in the laboratory, the germination and development of different genotypes of bean. The experiment was conducted at the Laboratory of the State University of Goiás, where were used ten cultivars of beans, subjected to germination in stress levels $0,-0.6,-1.2$ and $-1.8 \mathrm{MPa}$ osmotic potential, simulated with solutions of manitol $\left(\mathrm{C}_{6} \mathrm{H}_{14} \mathrm{O}_{6}\right)$ in levels: $0,44,58$, 89.17 and $133.75 \mathrm{~g} \mathrm{~L}^{-1}$. Among the genotypes used, cultivar Esplendor performed better for the variables analyzed, demonstrating high potential in adaptation to drought stress.

Key words: Phaseolus vulgaris L.; Germination; Stress. 


\section{Introdução}

O feijoeiro comum (Phaseolus vulgaris L.) é cultivado no Brasil o ano todo, em ecossistemas subtropical e tropical como Cerrado, Mata Atlântica e Semiárido, nos mais variados arranjos de plantas inter e intraespecíficos, em três safras: "das águas" e "da seca" em todos os estados da federação, e "de inverno", com irrigação concentrada nas Regiões Centro-Oeste, Sudeste e Oeste da Bahia, o que garante uma oferta constante do produto para um consumo interno ${ }^{1}$. Nas últimas décadas, têmse intensificado os debates sobre o aquecimento global e as mudanças climáticas, havendo quase um consenso entre os cientistas de que a intensificação do efeito estufa planetário encontra-se, principalmente, relacionada ao padrão de produção e ao consumo da sociedade moderna. No Brasil, alguns estudos mostram que elevação do nível do mar pode afetar regiões costeiras, especialmente, as metrópoles litorâneas e que os efeitos podem ser registrados em diversas outras regiões do país. Dentre os principais desequilíbrios que estas alterações podem causar ao meio ambiente, o Centro de Gestão e Estudos Estratégicos ${ }^{2}$ relaciona os seguintes: expansão de vetores de doenças endêmicas; mudança do regime hidrológico; perdas na agricultura e ameaças à biodiversidade e, claro, aumento da frequência e intensidade de enchentes e secas.

Nos últimos anos, os sistemas de produção agrícola convencionais veem cedendo espaço para os chamados sistemas alternativos de produção de base agroecológica. Fruto de um processo de conscientização ecológica em que a sociedade demonstra preferência, no qual o termo sustentabilidade se torna cada vez mais evidente ${ }^{3}$. Entretanto, a disponibilidade de água em algumas regiões do país é escassa e, algumas outras, estão passando por trocas climáticas bruscas, o que dificulta o plantio do feijão o ano todo ou mesmo na época das águas.

No entanto, o solo nem sempre oferece ambiente ótimo para a germinação das sementes, mesmo porque, quando ocorre deficiência hídrica, subentende-se que o solo apresenta potenciais hídricos que dificultam a absorção da água necessária para a germinação ${ }^{4}$. Neste caso, qualquer alteração, já no início de seu cultivo, irá influenciar o seu potencial produtivo. Portanto, os efeitos sobre a qualidade do produto, geralmente, são traduzidos pelo decréscimo na percentagem de germinação; aumento de plântulas anormais e redução do vigor das plântulas ${ }^{5}$, já na fase inicial de desenvolvimento destas.

A espécie possui pouca tolerância a estresses hídricos, sendo que, $60 \%$ do seu cultivo no mundo estão submetidos a este fator, tornando a seca o maior redutor da produtividade ${ }^{6}$. No entanto, algumas práticas de manejo podem contribuir para o aumento da produtividade, em condições de estresses hídricos, sendo que o maior progresso tem sido obtido por meio do melhoramento ${ }^{7}$. A utilização de cultivares tolerantes possibilita ao produtor a condição de manter sua produtividade em níveis que não causem dano econômico, expressos pela capacidade da planta em interligar uma diversidade de sinais ambientais a sinais metabólicos, que regulam a expressão gênica durante o estresse ${ }^{8}$, assim, possibilitando a sua sobrevivência. Segundo Santos ${ }^{9}$, é fundamental que ao analisar previamente, mediante levantamento de dados, o impacto que a seca e outras mudanças climáticas venham causar, seria possível realização de uma base de dados para trabalhar futuramente, contando com os possíveis imprevistos.

Uma das maneiras de sanar, em um curto período de tempo esse problema, seria a utilização de genótipos tolerantes que possam ser analisados e utilizados em programas de melhoramento como fonte de tolerância ao estresse hídrico e, assim, associar essa característica ou essas características aos demais genótipos que compõem os blocos de cruzamentos ${ }^{10}$. Alguns autores relatam que diversas soluções osmóticas têm sido usadas para simular um ambiente com reduzida umidade, dentre elas pode-se destacar o Manitol ${ }^{4}$.

Segundo Boyer ${ }^{5}$, os valores aproximados de potenciais de água letais, para algumas espécies vegetais, variam de $-1,4$ a $-6,0 \mathrm{MPa}$ para o tomateiro e a acácia, respectivamente. Entretanto, para o feijão-comum e para o feijão-caupi, esses valores de potencial hídrico foliar são de $-1,5$ a $-2,5 \mathrm{MPa}$, respectivamente, o que os classifica como plantas sensíveis e moderadamente tolerantes ao déficit de água no solo.

Diante do exposto e da importância econômica do feijão, torna-se imprescindível a realização de estudos, visando avaliar o desempenho de cultivares desenvolvidos para o cultivo em regime de sequeiro e irrigado, frente às limitações hídricas impostas durante 
o seu desenvolvimento. Assim, o presente trabalho teve como objetivo simular condições padronizadas de déficit hídrico, em laboratório, por meio de simulação com manitol, para a avaliação do desempenho fisiológico de diferentes cultivares de feijoeiro comum.

\section{Material e Métodos}

O experimento foi realizado no Laboratório da Universidade Estadual de Goiás, da Unidade Universitária de Ipameri (GO), no mês de novembro de 2012. Utilizaramse dez genótipos de feijão, provenientes do Programa de Melhoramento da Embrapa Arroz e Feijão (Pérola, Agreste, Esplendor, Campeiro, Cometa, Pontal, Marfim, Notável, Ametista e Estilo), os quais apresentavam características importantes, e são cultivados na região sudeste de Goiás, sendo estes submetidos à germinação, em condições de estresse hídrico sob os níveis $0,-0,6,-1,2$ e -1,8 MPa de potencial osmótico, simulados com soluções de manitol $\left(\mathrm{C}_{6} \mathrm{H}_{14} \mathrm{O}_{6}\right)$, nas doses: $0 ; 44 ; 58 ; 89,17$ e $133,75 \mathrm{~g} \mathrm{~L}^{-1}$. As concentrações foram calculadas pela fórmula de Van't Hoff: Yos = RTC, em que: Yos: potencial osmótico (atm); $\mathrm{R}$ : constante geral dos gases perfeitos $(0,082 \mathrm{~atm}$. L mol$\left.1^{\circ} \mathrm{K}^{-1}\right)$; T: temperatura $\left({ }^{\circ} \mathrm{C}\right)$ e $\mathrm{C}$ : concentração $\left(\mathrm{mol} \mathrm{L}^{-1}\right)$, conforme BRAGA ${ }^{12}$.

Foram colocadas 25 sementes de cada cultivar para germinar em três folhas de papel (Germitest), umedecidas com 2,25 vezes a sua massa, com as soluções osmóticas de manitol, para proporcionar diferentes níveis de potencial osmótico nas sementes, em delineamento inteiramente casualizado com quatro repetições. Logo após, depositadas sobre duas folhas e recobertas com uma terceira folha de papel. Em seguida, as folhas foram enroladas e depositadas em uma câmara de germinação (BOD), com umidade de $92 \%$ e temperatura de $25^{\circ} \mathrm{C}$, permanecendo nesta condição por um período de dez dias. A análise da germinação das sementes foi realizada no quinto dia e o crescimento das plântulas no décimo dia, na qual as avaliações, bem como a metodologia utilizada, obedeceram às normas estipuladas nas Regras para Análise de Sementes ${ }^{7}$. Posteriormente, para a análise do teste de geminação, foi utilizado o critério de emissão de radícula e, para o desenvolvimento, medição da radícula e do hipocótilo das plântulas normais desenvolvidas.

Foram submetidos os dados à análise de variância e comparação entre as médias pelo teste de Scott-Knott, a 5\% de significância, e, então, realizadas as análise de regressão polinomial para as diferentes concentrações de manitol, utilizando o programa SISVAR ${ }^{14}$. Além disso, realizou-se a análise de correlação de Spearman (r) para avaliar o grau de associação entre as doses de manitol e a porcentagem de germinação das sementes.

\section{Resultados e Discussão}

A análise de variância indicou diferença significativa entre as doses de manitol $(\mathrm{P}<0,05)$ e entre os genótipos avaliados $(\mathrm{P}<0,05)$, mas o efeito da interação entre as doses de manitol e os genótipos não foi significativo $(\mathrm{P}>$ $0,05)$, indicando que essas variáveis influenciaram de modo independente a germinação das sementes. As doses de manitol e o percentual de germinação apresentaram correlação negativa e significativa $(\mathrm{r}=-0,69 ; \mathrm{P}=0,00)$, indicando que a porcentagem de sementes germinadas diminuiu, à medida que o potencial osmótico foi reduzido (Tabela 1). Detecta-se que as sementes tiveram maior dificuldade para emitir radícula na condição de estresse hídrico simulado pelo Manitol. Estes resultados corroboram com os obtidos por Garcia ${ }^{15}$ em sementes de feijão e, por Trigo ${ }^{16}$, em sementes de cebola, os quais constataram redução significativa na percentagem de sementes germinadas com a redução do potencial osmótico.

O tratamento sem manitol (testemunha) proporcionou o maior percentual de germinação, no qual apresentou 97\%, seguido pelas doses 44 e $58 \mathrm{~g} \mathrm{~L}^{-1}$, com 88 e $91 \%$ de germinação, respectivamente. Por sua vez, a dose de $133 \mathrm{~g} \mathrm{~L}^{-1}$ proporcionou a menor quantidade de sementes germinadas (55\%), conforme Tabela 1.

Tabela 1. Porcentagem de germinação de sementes, utilizando dez cultivares de feijão comum, simulados com diferentes doses de solução de Manitol. Ipameri, GO, 2013.

\begin{tabular}{|c|c|}
\hline Doses (g L-1) & Porcentagem Germinação (\%) \\
\hline 0 & $97 \mathrm{a}$ \\
\hline 44 & $88 \mathrm{~b}$ \\
\hline 58 & $91 \mathrm{~b}$ \\
\hline 89 & $77 \mathrm{c}$ \\
\hline 133 & $55 \mathrm{~d}$ \\
\hline
\end{tabular}

Médias seguidas da mesma letra na coluna, não diferem entre si pelo teste de Scott-Knott a $5 \%$ de probabilidade. 
Avaliando diferentes níveis de potenciais osmóticos simulados por PEG-6000 em arroz, Silva ${ }^{17}$, encontrou resultados condizentes com o esperado e semelhantes ao apresentando por Manitol em feijão, neste trabalho, uma vez que a concentração de PEG-6000 e de Manitol controlam o meio de germinação e, dessa forma, a absorção de água pelos tecidos da semente, dificultando ou impedindo o início do processo germinativo. As diferentes concentrações das soluções e o tipo de produto utilizado para simular tais condições de estresse, podem alterar todo o resultado, mesmo se tratando de uma mesma cultivar, Coelho ${ }^{10}$ observou que ao utilizar manitol, $\mathrm{CaCl}_{2}, \mathrm{MgCl}_{2} \mathrm{e}$ $\mathrm{NaCl}$, em analogia às condições de campo, observou que a germinação reagiu de maneira diferenciada, dependente da concentração de cada soluto e o produto, sendo que a simulação por Manitol se mostrou mais estável e apresentou um maior potencial germinativo na mesma concentração dos demais testados. Entretanto, se o produto utilizado fosse $\mathrm{NaCl}$ e $\mathrm{CaCl}_{2}$, o potencial germinativo seria bem reduzido, mesmo na mesma concentração que o Manitol.

A Figura 1 ilustra a redução na porcentagem de sementes germinadas com a redução do potencial osmótico. Nota-se que da dose $0 \mathrm{~g} \mathrm{~L}^{-1}$ de manitol para a dose $133 \mathrm{~g} \mathrm{~L}^{-1}$ de manitol houve redução de $42 \%$ na porcentagem média de germinação das sementes.

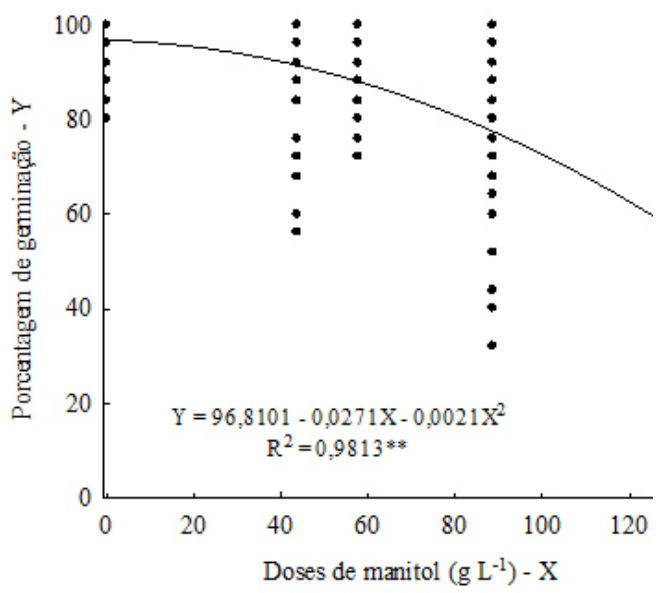

Figura 1. Análise de regressão para porcentagem de germinação de sementes de feijão comum, utilizando diferentes doses de Manitol. Ipameri, GO, 2013.
Neste sentido, Bray ${ }^{19}$ ressalta que a germinação das sementes é uma das fases mais sensíveis à deficiência hídrica, visto que a água constitui a matriz em que ocorre a maioria dos processos bioquímicos e fisiológicos, que resultam na protrusão da raiz primária. A diminuição do potencial osmótico prejudica a germinação, diminui o desenvolvimento meristemático e expõe as sementes por mais tempo às condições desfavoráveis do ambiente, acarretando diminuição na produtividade, como consequência, prejuízo ao produtor ${ }^{16,20}$.

Os cultivares BRS Esplendor e BRS Agreste apresentaram desempenho semelhante e superior aos demais cultivares testados, quanto à tolerância ao estresse hídrico, com o percentual médio de germinação de $96 \%$ e $90 \%$, respectivamente (Tabela 2). Por outro lado, os menores percentuais de germinação foram observados para os genótipos Cometa, Pontal, Pérola e Estilo, os quais apresentaram desempenho cerca de 23\% menor que o do cultivar Esplendor, demonstrando que possuem menor tolerância ao estresse hídrico. Além disso, esses percentuais podem indicar menor capacidade destes cultivares em interligar uma diversidade de sinais ambientais à sinais metabólicos que regulam a expressão gênica durante o estresse ${ }^{8}$, dificultando, assim, sua sobrevivência e impossibilitando uma boa produtividade.

Tabela 2. Porcentagem de germinação de dez cultivares de feijão comum, em relação às doses das soluções simuladoras utilizando Manitol. Ipameri, GO, 2013.

\begin{tabular}{|c|c|}
\hline CULTIVARES & $\begin{array}{c}\text { PORCENTAGEM } \\
\text { GERMINAÇÃO (\%) }\end{array}$ \\
\hline BRS ESPLENDOR & $96 \mathrm{a}$ \\
\hline BRS AGRESTE & $90 \mathrm{a}$ \\
\hline BRS AMETISTA & $85 \mathrm{~b}$ \\
\hline BRS CAMPEIRO & $84 \mathrm{~b}$ \\
\hline BRS MARFIM & $84 \mathrm{~b}$ \\
\hline BRS NOTÁVEL & $84 \mathrm{~b}$ \\
\hline BRS ESTILO & $79 \mathrm{c}$ \\
\hline BRS PÉROLA & $74 \mathrm{c}$ \\
\hline BRS PONTAL & $72 \mathrm{c}$ \\
\hline BRS COMETA & $70 \mathrm{c}$ \\
\hline MÉDIA GERAL & 81 \\
\hline
\end{tabular}

Médias seguidas da mesma letra na coluna, não diferem entre si pelo teste de Scott-Knott a $5 \%$ de probabilidade. 
O desenvolvimento de cultivares mais tolerantes a períodos de deficiência hídrica no solo, tal como o estudo do desenvolvimento de mecanismos que auxiliem as plantas a tolerar períodos prolongados de seca ou de estiagem, será essencial na manutenção da produção agrícola brasileira e mundial ${ }^{21}$. As diferentes reações de cada genótipo, a essas variações, permitem que este possa tolerá-las, quando estiverem níveis críticos no solo, ainda assim, mantendo suas taxas de fotossíntese em níveis adequados $^{22}$. Nesse caso, indicadas para a obtenção de novas famílias e geração de novos cultivares, além disso, possibilita a seleção de genótipos promissores de maneira precoce e, assim, acelerando o programa de melhoramento desta espécie.

Para a análise do desenvolvimento por meio da mensuração do comprimento radicular e comprimento do hipocótilo das plântulas de feijão, pode-se observar que houve diferença significativa $(\mathrm{P}<0,05)$ e, também, para interação dos genótipos com os diferentes níveis de potencial osmótico, indicando que essas variáveis influenciaram de modo dependente o desenvolvimento das plântulas. Nos maiores potenciais osmóticos, as plântulas são mais vigorosas, havendo maior desenvolvimento do sistema radicular com valores próximos a $12 \mathrm{~cm}$ e, à medida que se reduz o potencial osmótico, há redução do comprimento da raiz principal, corroborando com Reis ${ }^{23}$ e Moraes e Menezes ${ }^{20}$, que trabalharam com sementes de feijão e soja, respectivamente.

De acordo com a Tabela 3, é possível observar que o maior crescimento radicular ocorreu no nível 0 (zero) do soluto, para todos os genótipos. Cabe salientar, também, que houve redução no comprimento da radícula das plântulas para os dez genótipos avaliados, demonstrando que o aumento da concentração de manitol e redução no potencial hídrico afetou negativamente o desenvolvimento da radícula, causando uma diminuição no seu crescimento.

O cultivar esplendor destacou-se por apresentar radículas mais desenvolvidas quando expostas às maiores concentrações das soluções de manitol, indicando que este genótipo pode ser mais tolerante à deficiência hídrica que os outros cultivares testados. Ávila e colaboradores ${ }^{4}$ observaram que para a cultura da canola, contrariamente ao que ocorre com o feijão, quanto maior a dose de manitol, até o limite tolerável pela planta, maior é o crescimento radicular, pois, as plantas de canola, quando submetidas ao estresse hídrico, tendem a desenvolver um sistema radicular que propicia maior absorção água, em maiores profundidades. O uso eficiente da água pelas culturas agrícolas depende, sobretudo, das condições físicas do

Tabela 3. Crescimento radicular de dez cultivares de feijão comum, em diferentes doses de soluções simuladoras de estresse hídrico, utilizando manitol. Ipameri, GO, 2013.

\begin{tabular}{|c|c|c|c|c|c|}
\hline \multicolumn{7}{|c|}{ CRESCIMENTO RADÍCULA (cm) } \\
\hline \multirow{2}{*}{ GENÓTIPOS } & \multicolumn{5}{|c|}{ DOSES DE MANITOL } \\
\cline { 2 - 6 } & 0 & 44 & 58 & 89,17 & 133,75 \\
\hline BRS AGRESTE & $8,60 \mathrm{~b}$ & $9,20 \mathrm{a}$ & $6,71 \mathrm{~b}$ & $2,46 \mathrm{a}$ & $1,65 \mathrm{a}$ \\
\hline BRS AMETISTA & $7,42 \mathrm{c}$ & $8,95 \mathrm{a}$ & $6,57 \mathrm{~b}$ & $1,79 \mathrm{~b}$ & $1,34 \mathrm{a}$ \\
\hline BRS CAMPEIRO & $9,42 \mathrm{~b}$ & $6,70 \mathrm{c}$ & $4,57 \mathrm{c}$ & $1,38 \mathrm{~b}$ & $1,23 \mathrm{a}$ \\
\hline BRS COMETA & $8,87 \mathrm{~b}$ & $5,20 \mathrm{~d}$ & $3,40 \mathrm{~d}$ & $1,56 \mathrm{~b}$ & $2,26 \mathrm{a}$ \\
\hline BRS ESPLENDOR & $10,70 \mathrm{a}$ & $9,04 \mathrm{a}$ & $9,71 \mathrm{a}$ & $2,43 \mathrm{a}$ & $1,78 \mathrm{a}$ \\
\hline BRS ESTILO & $5,64 \mathrm{e}$ & $7,18 \mathrm{c}$ & $6,52 \mathrm{~b}$ & $2,37 \mathrm{a}$ & $1,30 \mathrm{a}$ \\
\hline BRS MARFIM & $7,76 \mathrm{c}$ & $7,80 \mathrm{~b}$ & $4,55 \mathrm{c}$ & $2,11 \mathrm{a}$ & $1,52 \mathrm{a}$ \\
\hline BRS NOTÁVEL & $9,51 \mathrm{~b}$ & $5,45 \mathrm{~d}$ & $6,12 \mathrm{~b}$ & $1,64 \mathrm{~b}$ & $1,43 \mathrm{a}$ \\
\hline BRS PÉROLA & $6,61 \mathrm{~d}$ & $5,75 \mathrm{~d}$ & $3,91 \mathrm{~d}$ & $1,49 \mathrm{~b}$ & $1,26 \mathrm{a}$ \\
\hline BRS PONTAL & $7,80 \mathrm{c}$ & $4,53 \mathrm{~d}$ & $3,30 \mathrm{~d}$ & $1,59 \mathrm{~b}$ & $1,14 \mathrm{a}$ \\
\hline MÉDIAS & $\mathbf{8 , 2 1}$ & $\mathbf{7 , 0 0}$ & $\mathbf{5 , 5 3}$ & $\mathbf{1 , 8 8}$ & $\mathbf{1 , 4 9}$ \\
\hline
\end{tabular}

Médias seguidas da mesma letra na coluna, não diferem entre si pelo teste de Scott-Knott a $5 \%$ de probabilidade. 
solo; das condições atmosféricas; do estado nutricional das plantas; de fatores fisiológicos; da natureza genética e do seu estágio de desenvolvimento. Todas as plantas exigem quantidades relativamente elevadas de água para a produção de matéria seca, uma cultura de feijão, por exemplo, requer cerca de $1750 \mathrm{~kg}$ de água para a produção de $1 \mathrm{~kg}$ de matéria seca e grãos ${ }^{25}$, se o cultivar necessitar de uma menor quantidade de água, além da tolerância, irá acarretar menor custo de produção e em um cultivo mais sustentável.

Os cultivares que apresentaram sensibilidade à deficiência hídrica foram o BRS Pontal e BRS Cometa, os quais, nas concentrações mais altas, apresentaram redução do crescimento radicular, quando comparados aos demais. Na dose de 133,75\% não houve diferença entre os cultivares testados, demonstrando que neste potencial hídrico, as plântulas não conseguem absorver água e, consequentemente, não apresentam diferença em seu crescimento vegetativo que seja significativo.

Da mesma forma, para o comprimento do hipocótilo, pode ser verificada redução nesta característica com a redução do potencial osmótico (Tabela 4). Corroborando os resultados de $\mathrm{Reis}^{23}$, Moraes e Menezes $^{20}$, que trabalharam com sementes de feijão e soja, respectivamente. Em ambos os casos, os menores potenciais osmóticos reduziram o comprimento das plântulas, devido às mudanças na turgescência celular, em função da diminuição da síntese de proteínas em condições de déficit hídrico. O primeiro efeito mensurável do estresse hídrico é uma diminuição no crescimento, causada pela redução da expansão celular que necessita de potencial de turgor adequado ${ }^{26}$.

Os resultados encontrados estão de acordo com os obtidos por Alvarenga ${ }^{27}$, em estudos realizados com soja, e $\operatorname{Dantas}^{28}$ em estudos com feijão, em que à medida que aumentou a concentração de manitol nas soluções, houve decréscimo no comprimento do hipocótilo das plantas, demonstrando ter seu desenvolvimento prejudicado quando submetidos a ambientes com baixa umidade. Para este caráter o Cultivar BRS Esplendor também apresentou indícios de ser o mais adaptado às condições de deficiência de água, pois, alcançou os maiores valores para o comprimento do hipocótilo em concentrações mais elevadas de manitol (58\%), assim como o agreste (Tabela 4).

\section{Conclusão}

A redução do potencial osmótico do substrato, utilizando manitol, pode ser utilizada para o estudo de

Tabela 4. Crescimento do hipocótilo dos diferentes genótipos em relação às doses das soluções simuladoras de estresse hídrico, utilizando manitol. Ipameri, GO, 2013.

\begin{tabular}{|c|c|c|c|c|c|}
\hline \multicolumn{6}{|c|}{ CRESCIMENTO HIPOCÓTILO (CM) } \\
\hline \multirow{2}{*}{ CULTIVARES } & \multicolumn{5}{|c|}{ DOSES (\%) } \\
\hline & 0 & 44 & 58 & 89,17 & 133,75 \\
\hline BRS AGRESTE & $6,99 \mathrm{~b}$ & $4,15 \mathrm{~d}$ & $3,17 \mathrm{a}$ & $0,31 \mathrm{a}$ & $0,08 \mathrm{a}$ \\
\hline BRS AMETISTA & $5,37 \mathrm{~d}$ & $4,96 \mathrm{a}$ & $2,47 \mathrm{~b}$ & $0,20 \mathrm{a}$ & $0,07 \mathrm{a}$ \\
\hline BRS CAMPEIRO & $9,07 \mathrm{a}$ & $2,84 \mathrm{c}$ & $2,56 \mathrm{~b}$ & $0,30 \mathrm{a}$ & $0,05 \mathrm{a}$ \\
\hline BRS COMETA & $8,79 \mathrm{a}$ & $2,46 \mathrm{e}$ & $2,85 \mathrm{~b}$ & $0,42 \mathrm{a}$ & $0,07 \mathrm{a}$ \\
\hline BRS ESPLENDOR & $9,11 \mathrm{a}$ & $3,19 \mathrm{c}$ & $3,71 \mathrm{a}$ & $0,58 \mathrm{a}$ & $0,06 \mathrm{a}$ \\
\hline BRS ESTILO & $4,82 \mathrm{e}$ & $2,92 \mathrm{c}$ & $2,33 \mathrm{~b}$ & $1,01 \mathrm{a}$ & $0,13 \mathrm{a}$ \\
\hline BRS MARFIM & $4,50 \mathrm{e}$ & $3,14 \mathrm{c}$ & $2,52 \mathrm{~b}$ & $0,48 \mathrm{a}$ & $0,19 \mathrm{a}$ \\
\hline BRS NOTÁVEL & $6,58 \mathrm{~b}$ & $2,11 \mathrm{e}$ & $1,20 \mathrm{~d}$ & $0,30 \mathrm{a}$ & $0,01 \mathrm{a}$ \\
\hline BRS PÉROLA & $5,88 \mathrm{c}$ & $2,90 \mathrm{c}$ & $2,52 \mathrm{~b}$ & $0,41 \mathrm{a}$ & $0,19 \mathrm{a}$ \\
\hline BRS PONTAL & $6,99 \mathrm{c}$ & $3,84 \mathrm{~b}$ & $2,01 \mathrm{c}$ & $0,22 \mathrm{a}$ & $0,09 \mathrm{a}$ \\
\hline MÉDIAS & 6,72 & 3,24 & 2,53 & 0,41 & 0,18 \\
\hline
\end{tabular}

Médias seguidas da mesma letra na coluna, não diferem entre si pelo teste de Scott-Knott a $5 \%$ de probabilidade. 
possíveis genótipos com maior tolerância à deficiência hídrica. Dentre os cultivares testados, o cultivar BRS Esplendor teve melhor desempenho para a maioria dos caracteres avaliados, demonstrando maior potencial adaptativo à deficiência hídrica, característica importante para os programas de melhoramento.

\section{Referências}

1. Albuquerque, A. C. S.; Silva, A. G. da. Embrapa Informação Tecnológica, 2008, 1, 219.

2. CGEE. Centro de Gestão e Estudos Estratégicos. Manual de Capacitação sobre a Mudança do Clima e Projetos de Mecanismo de Desenvolvimento Limpo, 2008, 1, 1.

3. Santos, J. O. D.; Santos, R. M. D. S.; Fernandes, A. D. A.; Souso, J. D. S.; Borges, M. D. G. B.; Ferreira, R. T. F. V.; Salgado, A. B. Agropecuária científica no semiárido, 2013, 9, 09.

4. Machado Neto, N. B.; Custódio, C. C.; Costa, P. R.; Doná, F. L. Revista Brasileira de Sementes, 2006, 28, 142.

5. Toledo, M. Z.; Fonseca, N. R.; César, M. L.; Soratto, R. P.; Cavariani, C.; Crusciol, C. A. C. Pesquisa Agropecuária Tropical, 2009, 39, 124.

6. Aguiar, R. S.; Moda-Cirino, V.; Faria, R. T.; Vidal, L. H. I. Semina, 2008, 29, 1.

7. Singh, S. P. Crop Science Society of America, 1995, 35, 118.

8. Custódio, C. C.; Salomão, G. R.; Neto, N. B. M. Revista Ciência Agronômica, 2009, 40, 617.

9. Santos, R. S. dos; Costa, L. C.; Sediyama, G. C.; Leal, B. G.; Oliveira, R. A. de; Justino, F. B. Revista Brasileira de meteorologia, 2011, 26, 314.

10. Vale, N. M.; Barili, L. D.; Rozzeto, D. S.; Stinghin, J. C.; Coimbra, J. L. M.; Guidolin, A. F.; Koop, M. M. Biotemas, 2012, 25, 135.

11. Boyer, J. S. Academic Press, 1978, 4, 154.

12. Braga, L. F.; Sousa, M. P.; Braga, J. F.; Sá, M. E. Revista Brasileira de Sementes, 1999, 21, 95.

13. Brasil. Ministério da Agricultura. Regras para análises de sementes. 2009, $1,1$.

14. Ferreira, D.F. Revista Symposium, 2008, 6, 36.

15. Garcia, S. H.; Rozzetto, D. S.; Coimbra, J. L. M.; Guidolin, A. F. Revista de Ciências Agroveterinárias, 2012, 11, 35.

16. Trigo, M.F.O.O.; Trigo L.F.N. Revista Brasileira de Sementes, 1999, $21,145$.

17. Silva, L. S.; Silva, R. B.; Valadares, R. N.; Matos, V. P.; Lima, C. F. Revista Tropica, 2012, 6, 37.
18. Coelho, D. L. M.; Agostini, E. A. T. D.; Guaberto, L. M.; Machado-Neto, N. B.; Custódio, C. C. Acta Scientiarum. Agronomy, 2010, 32, 491.

19. Bray, C. F. Seed development and germination, 1995, 1, 767.

20. Moraes, G.A.F.; Menezes, N.L. Ciência Rural, 2003, 33, 219.

21. Nepomuceno, A. L.; Neumaier, N.; Farias, J. R. B.; Oya, T. Revista Biotecnologia, Ciência \& Desenvolvimento, 2001, 1, 12.

22. Ribeiro, R. V.; Santos, M. G.; Souza, G. M.; Machado, E. C.; Oliveira, R. F. Angelocci, L. R. Pimentel, C. Pesquisa Agropecuária Brasileira, 2004, 39, 615.

23. Reis, I.S. Informativo ABRATES, 2003, 13, 89.

24. Ávila, M. R.; Braccini, A. D. L.; Scapim, C. A.; Fagliari, J. R.; Santos, J. D. Revista Brasileira de Sementes, 2007, 29, 98.

25. Doorenbos J.; Kassam, A.H. Yield response to water, 1979, 1, 201.

26. Taiz, L. Zeiger, E. Fisiologia vegetal, 2004, 3, 719.

27. Alvarenga, E.M. Revista Brasileira de Sementes, Brasília, 1991, $13,189$.

28. Dantas, B. F.; Ribeiro, L. D. S.; Aragão, C. A. Revista Brasileira de Sementes, 2007, 29,106.

\section{Daiane M. Duarte', Diana C. da Silva', Ednaldo C. Rocha², Helton S. Pereira ${ }^{3} \&$ Fabrício Rodrigues ${ }^{2 *}$}

\footnotetext{
${ }^{1}$ Universidade Estadual de Goiás, Departamento de Produção Vegetal, Rodovia GO 330, Km 241, Anel Viário, s/n, Setor Universitário, CEP 75780-000, Ipameri-GO, Brasil.

${ }^{2}$ Universidade Estadual de Goiás, Departamento de Agronomia e Engenharia Florestal, Rodovia GO 330, Km 241, Anel Viário, s/n, Setor Universitário, CEP 75780-000, Ipameri-GO, Brasil.

${ }^{3}$ Embrapa arroz e feijão, Departamento de Melhoramento de feijão, Goiânia, GO, Embrapa Arroz e Feijão, Rodovia GO-462,(GoiâniaNova Veneza), km 12, Zona Rural, C.P. 179, 75375-000, Santo Antônio de Goiás, GO, Brasil.

*e-mail: fabricio.rodrigues@ueg.br
} 
